\title{
A BOUNDARY VALUE PROBLEM FOR A SINGULARLY PERTURBED DIFFERENTIAL EQUATION
}

\section{S. HABER AND N. LEVINSON}

The two point boundary value problem $\left({ }^{\prime}=d / d x\right)$

$$
\epsilon y^{\prime \prime}=f\left(x, y, y^{\prime}, \epsilon\right), \quad y(0)=a, \quad y(1)=b
$$

for small $\epsilon$ will be solved in the case where the degenerate equation

$$
f\left(x, u, u^{\prime}, 0\right)=0
$$

has a solution $u=g(x)$ for $0 \leqq x \leqq x_{0}$ with $g(0)=a$ and $u=h(x)$ for $x_{0} \leqq x \leqq 1$ with $h(1)=b$ where $g\left(x_{0}\right)=h\left(x_{0}\right)$. It will be assumed that $g^{\prime}\left(x_{0}\right) \neq h^{\prime}\left(x_{0}\right)$.

The case of (1) with $f=1-\left(y^{\prime}\right)^{2}$ and where $|a-b|<1$ can be treated explicitly. For small $\epsilon>0$ the solution of (1) tends to the broken line solution of (2) with $g(x)=a-x$ and $h=b-1+x$ and $x_{0}=(1+a-b) / 2$. (There is another broken line solution of (2) with $g=a+x$ and $h=b+1-x$ but there is no solution of (1) for $\epsilon>0$ near this broken line solution of (2). As will be seen the criteria below will single out only the first broken line solution.)

To formulate the general problem more precisely let $y_{0}=g\left(x_{0}\right)$ $=h\left(x_{0}\right)$ and let $\mu_{1}=g^{\prime}\left(x_{0}\right), \mu_{2}=h^{\prime}\left(x_{0}\right)$. The case $\mu_{2}>\mu_{1}$ will be considered here. (The case $\mu_{1}<\mu_{2}$ can be treated in the same way or can be reduced to the first case by replacing $y$ by $-y$.) Let $\alpha, \beta$, and $\epsilon_{0}$ be positive constants. Let $U(x)=g(x)$ for $0 \leqq x \leqq x_{0}$ and let $U(x)=h(x)$ for $x_{0} \leqq x \leqq 1$. Let $R$ be the region of $(x, y, w, \epsilon)$ space determined by $0 \leqq \epsilon \leqq \epsilon_{0}$, and by the union of

$$
0 \leqq x<x_{0}<x \leqq 1, \quad|y-U(x)| \leqq \alpha, \quad\left|w-U^{\prime}(x)\right| \leqq \beta
$$

and

$$
\left|x-x_{0}\right| \leqq \alpha, \quad\left|y-y_{0}\right| \leqq \alpha, \quad \mu_{1}-\beta \leqq w \leqq \mu_{2}+\beta .
$$

In $R$ let $f(x, y, w, \epsilon)$ be real-valued and of class $C$. Moreover let $f_{y}(=\partial f / \partial y)$ and $f_{w}$ exist and be of class $C$.

With no loss in generality it may be assumed that

$$
\mu_{1}<0<\mu_{2}
$$

since replacing $y$ by $\tilde{y}+\left(\mu_{1}+\mu_{2}\right) x / 2$ will achieve this.

THEOREM I. Let $f(x, y, w, \epsilon), f_{\nu}$ and $f_{w}$ be of class $C$ in $R$ and let the

Received by the editors September 6, 1954 and, in revised form, January 24, 1955. 
degenerate system (2) have the solutions $g(x)$ and $h(x)$ over $\left[0, x_{0}\right]$ and $\left[x_{0}, 1\right]$ respectively as described above. Let

$$
\begin{array}{ll}
f_{w}\left(x, g(x), g^{\prime}(x), 0\right)>0, & 0 \leqq x \leqq x_{0}, \\
f_{w}\left(x, h(x), h^{\prime}(x), 0\right)<0, & x_{0} \leqq x \leqq 1 .
\end{array}
$$

Further let

$$
f\left(x_{0}, y_{0}, w, 0\right)>0, \quad \mu_{1}<w<\mu_{2} .
$$

Then for sufficiently small $\epsilon>0$ there exists a solution of the boundary value problem (1)

$$
y=\Phi(x, \epsilon)
$$

such that

$$
\left(x, \Phi(x, \epsilon), \Phi^{\prime}(x, \epsilon), \epsilon\right) \in R \quad \text { for } 0 \leqq x \leqq 1 .
$$

Moreover as $\epsilon \rightarrow 0+, \Phi(x, \epsilon) \rightarrow U(x)$ uniformly over $[0,1]$ and $\Phi^{\prime}(x, \epsilon)$ $\rightarrow U^{\prime}(x)$ uniformly over $\left[0, x_{0}-\delta_{0}\right]$ and $\left[x_{0}+\delta_{0}, 1\right]$ for any fixed $\delta_{0}>0$.

THEOREM II. The solution $\Phi(x, \epsilon)$ of (1) is unique in that given an $\omega>0$ there exists $\delta_{1}>0$ such that for small $\epsilon>0$ there is no solution $\Psi(x, \epsilon)$ of $(1)$ other than $\Phi(x, \epsilon)$ which satisfies

$$
|\Psi(x, \epsilon)-U(x)|<\delta_{1}, \quad\left|x-x_{0}\right|<\omega .
$$

The solution $u=h(x)$ of (2) can be continued to the left over some interval since

$$
f_{w}\left(x_{0}, y_{0}, \mu_{2}, 0\right) \neq 0 .
$$

Thus there is a $\delta>0$ such that $h(x)$ is a solution of (2) for $x_{0}-\delta \leqq x \leqq 1$ and similarly $g(x)$ is a solution of (2) for $0 \leqq x \leqq x_{0}+\delta$. Let $\rho$ be a region of $(\xi, \eta)$ space determined by

$$
\left|x_{0}-\xi\right| \leqq \delta, \quad|\eta-h(\xi)| \leqq \Delta
$$

where $\Delta$ will be specified later.

It will be shown that there is an $\epsilon_{1}>0$ such that for $0<\epsilon<\epsilon_{1}$ and for $(\xi, \eta) \in \rho$ there is a solution $y=\phi(x, \xi, \eta, \epsilon)$ of

$$
\epsilon y^{\prime \prime}=f\left(x, y, y^{\prime} \epsilon\right), \quad y(\xi)=\eta, y^{\prime}(\xi)=0,
$$

for $\xi \leqq x \leqq 1$. Moreover $\left(x, \phi, \phi^{\prime}, \epsilon\right) \in R$. It will also be shown that for each $\xi$ there is a unique $\eta=F(\xi),(\xi, F(\xi)) \in \rho$, such that

$$
\phi(1, \xi, F(\xi), \epsilon)=b \text {. }
$$

(Of course $F$ also depends on $\epsilon$.) It will be shown that $F$ is differ- 
entiable and $F^{\prime}>0$ and that as $\epsilon \rightarrow 0, F(\xi) \rightarrow h(\xi),\left|\xi-x_{0}\right| \leqq \delta$.

In the same way a curve $\eta=G(\xi)$ exists such that (5) has a solution $\tilde{\phi}(x, \xi, G(\xi), \epsilon), 0 \leqq x \leqq \xi$, and $\tilde{\phi}(0, \xi, G, \epsilon)=a$. Moreover $G^{\prime}(\xi)<0$ and $G(\xi) \rightarrow g(\xi)$ over $\left|\xi-x_{0}\right| \leqq \delta$ as $\epsilon \rightarrow 0$. Since $y=g(x)$ and $y=h(x)$ intersect at $x=x_{0}$ it follows that, for small $\epsilon, \eta=G(\xi)$ and $\eta=F(\xi)$ must intersect for $\left|\xi-x_{0}\right| \leqq \delta$ and because $F^{\prime}>0$ and $G^{\prime}<0$ the point of intersection is unique. The solution of $\epsilon y^{\prime \prime}=f, 0 \leqq x \leqq 1$ with zero slope at the point of intersection is clearly a solution of (1). Thus the proof of Theorem I has been made to depend on the proof that $F$ (and $G$ ) exist and have the properties described above.

Proof of the existence of $\phi(x, \xi, \eta, \epsilon)$. Because $f\left(x, h, h^{\prime}, 0\right)=0$ and $f_{w}\left(x, h, h^{\prime}, 0\right)<0$ it follows that for $1 \geqq x \geqq x_{0}-\delta$ and $|u-h| 4\left|w-h^{\prime}\right|$ small there is a unique solution $w=J(x, u)$ of $f(x, u, w, 0)=0$ and $J$ and $J_{u}$ are of class $C$. Since $u^{\prime}=J(x, u)$ has $u=h(x)$ as a solution over $\left[x_{0}-\delta, 1\right]$ it follows that if $\Delta$ is small, then for any $(\xi, \eta)$ of $\rho$ there is a solution $u=\psi(x, \xi, \eta)$ of $u^{\prime}=J(x, u)$ over $\left[x_{0}-\delta, 1\right]$ or that

$$
f\left(x, u, u^{\prime}, 0\right)=0, \quad u(\xi)=\eta,
$$

has a solution $\psi(x, \xi, \eta)$ over $\left[x_{0}-\delta, 1\right]$. Moreover because $J_{u}$ is bounded, $\psi(x, \xi, \eta) \rightarrow h(x)$ and $\psi^{\prime} \rightarrow h^{\prime}$ as $\eta \rightarrow h(\xi)$, and because $J_{u}$ is continuous, $\partial \psi / \partial \eta$ exists and is positive. Thus $\psi(1, \xi, \eta)$ is monotone increasing in $\eta$. For $\eta>h(\xi), \psi(1, \xi, \eta)>b$ and similarly for $\eta<h(\xi)$, $\psi(1, \xi, \eta)<b$.

The solution of (5), $\phi(x, \xi, \eta, \epsilon)$, certainly exists for $x$ near $\xi$. It will be shown that for $(\xi, \eta) \in \rho,\left(x, \phi, \phi^{\prime}, \epsilon\right) \in R$ as $x$ increases so that $\phi$ can be continued to $x=1$.

It follows from (4) and the continuity of $f$ that given any $\gamma>0$ there is a $k_{0}>0$ and $\delta^{\prime}>0$ such that for $\left|x-x_{0}\right|+\left|y-y_{0}\right|<\delta^{\prime}$,

$$
f(x, y, w, 0)>k_{0}, \quad \mu_{1}+\gamma<w<\mu_{2}-\gamma .
$$

It is also the case that (3) and the continuity of $f_{w}$ imply the existence of a $\delta^{\prime \prime}>0$ and $k>0$ such that

$$
f_{w}(x, y, w, 0)<-k, \quad|y-h(x)|+\left|w-h^{\prime}(x)\right| \leqq \delta^{\prime \prime}
$$

for $x_{0}-\delta \leqq x \leqq 1$.

The notation $\theta(\epsilon)$ will be used to denote a continuous function of $\epsilon$ for $0 \leqq \epsilon<\epsilon_{0}$ with $\theta(0)=0$. The function $\theta$ will be used in the generic sense and may be different in each formula. The generic constant $A$, which will depend only on $f(x, y, w, \epsilon)$, in $R$, will also be used. Let $\gamma<\delta^{\prime \prime} / 10$. Using (6) it follows that so long as $\left|x-x_{0}\right|+\left|\phi-y_{0}\right|<\delta^{\prime}, \epsilon$ is small, and $0 \leqq \phi^{\prime} \leqq \mu_{2}-\gamma$

$$
\epsilon \boldsymbol{\phi}^{\prime \prime}=f\left(\boldsymbol{x}, \boldsymbol{\phi}, \boldsymbol{\phi}^{\prime}, \boldsymbol{\epsilon}\right)>k_{0} / 2 .
$$


Integrating the inequality yields $\epsilon \phi^{\prime}(x, \xi, \eta, \epsilon)>k_{0}(x-\xi) / 2$ or

$$
x-\xi<2 \epsilon \mu_{2} / k_{0} \text {. }
$$

Since

$$
|\phi(x, \xi, \eta, \epsilon)-\eta| \leqq \max \left|\phi^{\prime}\right|(x-\xi) \leqq 2 \epsilon \mu_{2}^{2} / k_{0}
$$

it follows that if $\epsilon$ is small, $\Delta$ small, and $\delta$ decreased if necessary, then $\phi^{\prime}(x, \xi, \eta, \epsilon)$ first becomes $\mu_{2}-\gamma$ for some $x_{1}<\xi+2 \epsilon \mu_{2} / k_{0}$.

Let $z=\phi(x, \xi, \eta, \epsilon)-\psi(x, \xi, \eta)$. Then

$$
\epsilon z^{\prime \prime}=f\left(x, \phi, \phi^{\prime}, \epsilon\right)-f\left(x, \psi, \psi^{\prime}, 0\right)-\epsilon \psi^{\prime \prime} .
$$

For $\xi \leqq x \leqq x_{1},|\psi(x, \xi, \eta)-\eta| \leqq A\left(x_{1}-\xi\right)=A \epsilon$ and

$$
\begin{aligned}
\left|\psi^{\prime}(x, \xi, \eta)-\mu_{2}\right| & \leqq\left|\psi^{\prime}(x, \xi, \eta)-h^{\prime}(x)\right|+\left|h^{\prime}(x)-\mu_{2}\right| \\
& \leqq A|\eta-h(\xi)|+A \epsilon \leqq A(\epsilon+\Delta) .
\end{aligned}
$$

Thus at $x=x_{1}$

$$
|z| \leqq A \epsilon, \quad\left|z^{\prime}\right| \leqq \gamma+A(\epsilon+\Delta) .
$$

Using (9) and the mean value theorem

$$
\epsilon z^{\prime \prime}=P z^{\prime}+Q z+S
$$

where $|S| \leqq \theta(\epsilon),|Q| \leqq A$ and by (7)

$$
P \leqq-k
$$

so long as

$$
|\phi-h|+\left|\phi^{\prime}-h^{\prime}\right| \leqq \delta^{\prime \prime} .
$$

Choose $\Delta$ small enough so that

$$
|\psi-h|+\left|\psi^{\prime}-h^{\prime}\right| \leqq \delta^{\prime \prime} / 2 \text {. }
$$

From (11)

$$
\begin{aligned}
z^{\prime}(x) & =z^{\prime}\left(x_{1}\right) \exp \left(\int_{x_{1}}^{x} P(s) d s / \epsilon\right) \\
& +\frac{1}{\epsilon} \int_{x_{1}}^{x}(Q z+S) \exp \left(\int_{t}^{x} P(s) d s / \epsilon\right) d t
\end{aligned}
$$

From (10) and (12) for $x \geqq x_{1}$ (and so long as (13) holds)

$$
\begin{gathered}
\left|z^{\prime}(x)\right| \leqq \\
+\gamma+A(\epsilon+\Delta)] \exp \left(-k\left(x-x_{1}\right) / \epsilon\right)+\theta(\epsilon) \\
+\frac{A}{\epsilon} \int_{x_{1}}^{x}|z(t)| \exp (-k(x-t) / \epsilon) d t .
\end{gathered}
$$


Integrating (15) and using (10) there follows for $x \geqq x_{1}$

$$
|z(x)| \leqq \theta(\epsilon)+A \int_{x_{1}}^{x}|z(t)| d t
$$

This implies

$$
\int_{x_{1}}^{x}|z(t)| d t \leqq \frac{\theta(\epsilon)}{A}\left(\exp \left(A\left(x-x_{1}\right)\right)-1\right)
$$

or

$$
|z(x)| \leqq \theta(\epsilon) e^{A}=\theta(\epsilon)
$$

In (15) this gives

$$
\left|z^{\prime}(x)\right| \leqq(\gamma+A \Delta) \exp \left(-K\left(x-x_{1}\right) / \epsilon\right)+\theta(\epsilon) .
$$

Thus if $\epsilon$ and $\Delta$ are small enough then (16) and (17) with (14) show that (13) will hold for $x_{1} \leqq x \leqq 1$. Thus $\phi$ exists for $\xi \leqq x \leqq 1$ and from the derivation above, (10) and (16), and (17),

$$
\begin{array}{ll}
\left|f_{w}\left(x, \phi, \phi^{\prime}, \epsilon\right)\right| \leqq A, & \xi \leqq x \leqq x_{1}, \\
\left|f_{y}\left(x, \phi, \phi^{\prime}, \epsilon\right)\right| \leqq A, & \xi \leqq x \leqq 1,
\end{array}
$$

and using (7)

$$
f_{w}\left(x, \phi, \phi^{\prime}, \epsilon\right) \leqq-k / 2, \quad x_{1} \leqq x \leqq 1 .
$$

Proof that $\partial \phi / \partial \eta>0$. Because $f_{y}$ and $f_{w}$ exist, $w(x)=\partial \phi / \partial \eta$ exists. It will be shown that $w(x) \geqq 0$. Clearly

$$
w(\xi)=1, \quad w^{\prime}(\xi)=0,
$$

and

$$
\epsilon w^{\prime \prime}-f_{w}\left(x, \phi, \phi^{\prime}, \epsilon\right) w^{\prime}-f_{v} w=0 .
$$

Transposing $f_{y} w$ and integrating the left side by using the usual integrating factor there follows

$$
\left|w^{\prime}(x)\right| \leqq \frac{A}{\epsilon} \int_{\xi}^{x}|w(s)| \exp (A(x-s) / \epsilon) d s .
$$

For $\xi \leqq x \leqq x_{1}$ there follows since $x_{1}<\xi+A \epsilon$

$$
\left|w^{\prime}(x)\right| \leqq \frac{A}{\epsilon} \int_{\xi}^{x}|w(s)| d s .
$$

Thus so long as $|w| \leqq 2$ 


$$
\left|w^{\prime}(x)\right| \leqq A,
$$

But

$$
|w(x)-1| \leqq \int_{\xi}^{x}\left|w^{\prime}(s)\right| d s \leqq A \epsilon, \quad \xi \leqq x \leqq x_{1} .
$$

For $x \geqq x_{1}$ let

$$
w=\exp \left(-\lambda\left(x-x_{1}\right) / \epsilon\right) p
$$

where the constant $\lambda$ will be chosen later. Then

$$
\begin{aligned}
w^{\prime} & =\exp \left(-\lambda\left(x-x_{1}\right) / \epsilon\right)\left(p^{\prime}-\frac{\lambda}{\epsilon} p\right), \\
w^{\prime \prime} & =\exp \left(-\lambda\left(x-x_{1}\right) / \epsilon\right)\left(p^{\prime \prime}-\frac{2 \lambda}{\epsilon} p^{\prime}+\frac{\lambda^{2}}{\epsilon^{2}} p\right) .
\end{aligned}
$$

In (21) this gives

$$
\epsilon p^{\prime \prime}-\left(2 \lambda+f_{w}\right) p^{\prime}+\left(\frac{\lambda^{2}+\lambda f_{w}}{\epsilon}-f_{y}\right) p=0 .
$$

Choose $\lambda=k / 4$ so by (20)

$$
\lambda^{2}+\lambda f_{w}<-k^{2} / 16
$$

From (22) and (23) it follows that

$$
p\left(x_{1}\right)>1 / 2
$$

and thus for small $\epsilon$

$$
p^{\prime}\left(x_{1}\right)>0 \text {. }
$$

By (25) the coefficient of $p$ in (24) is negative. Thus $p$ which starts out positive increasing can never have $p^{\prime}=0$ and so $p$ is increasing over $\left[x_{1}, 1\right]$ and therefore positive. Thus $w$ is positive, that is

$$
\frac{\partial \phi}{\partial \eta}(x, \xi, \epsilon)>0, \quad \xi \leqq x \leqq 1 .
$$

Proof of the existence of $F(\xi)$. It will next be shown that for any $\xi,\left|\xi-x_{0}\right|<\delta$, there is a unique $\eta=F(\xi),|\eta-h(\xi)|<\Delta$, such that

$$
\phi(1, \xi, F(\xi), \epsilon)=b .
$$

Indeed let $\eta_{1}=h(\xi)-\Delta / 2$. Then $\psi\left(1, \xi, \eta_{1}\right)<b$. Because of (16) it follows that for small $\epsilon$

$$
\phi\left(1, \xi, \eta_{1}, \epsilon\right)<b .
$$


Similarly for $\eta_{2}=h(\xi)+\Delta / 2, \phi\left(1, \xi, \eta_{2}, \epsilon\right)>b$. Since $\phi(1, \xi, \eta, \epsilon)$ is continuous in $\eta$ and because of (26) the existence of a unique $F(\xi)$ follows. Because $\partial \phi / \partial \eta \neq 0$ and is continuous it follows that $F(\xi)$ is a continuous function of $\xi$ (and in fact of $(\xi, \epsilon)$ ).

Because $\partial \phi / \partial \xi$ also exists and is continuous it follows from (27) that $F^{\prime}(\xi)$ exists and is given by

Let

$$
F^{\prime}(\xi)=-\frac{\partial \phi}{\partial \xi}(1, \xi, F(\xi), \epsilon) / \frac{\partial \phi}{\partial \eta}(1, \xi, F(\xi), \epsilon) .
$$

$$
v(x)=\frac{\partial \phi}{\partial \xi}(x, \xi, \eta, \epsilon) .
$$

Then it is easily seen, since $\phi(\xi, \xi, \eta, \epsilon)=\eta$ and $\phi^{\prime}(\xi, \xi, \eta, \epsilon)=0$, that

$$
v(\xi)=0, \quad v^{\prime}(\xi)=-f(\xi, \eta, 0, \epsilon) / \epsilon<0 .
$$

Now $v(x)$ is a solution of (21). Clearly $w$ and $v$ are independent solutions of (21) and thus the Wronskian

$$
J(x)=w^{\prime}(x) v(x)-w(x) v^{\prime}(x)
$$

is nonvanishing. From (29) and $w(\xi)=1$ it follows that $J(\xi)>0$ so that $J(x)>0$. Consider now

$$
\frac{d}{d x} \frac{-\frac{\partial \phi}{\partial \xi}(x, \xi, \eta, \epsilon)}{\frac{\partial \phi}{\partial \eta}(x, \xi, \eta, \epsilon)}=-\frac{d}{d x} \frac{v}{w}=\frac{J(x)}{w^{2}}>0 .
$$

Since at $x=\xi, v / w=0$ it follows from (30) that at $x=1,-v / w>0$. In (28) this makes

$$
F^{\prime}(\xi)>0 .
$$

As was shown below (5) this proves Theorem I.

The proof of Theorem II follows easily also. Indeed let $\Psi(x, \epsilon)$ be a solution of (1) different from $\Phi(x, \epsilon)$ and satisfying

$$
|\Psi(x, \epsilon)-U(x)|<\delta_{1}, \quad\left|x-x_{0}\right|<\omega .
$$

Because $\Psi$ is close to $U$ and because $U^{\prime}\left(x_{1}-0\right)<0$ and $U^{\prime}\left(x_{1}+0\right)>0$ it is clear that given any $\delta_{2}>0$ then, if $\delta_{1}$ is chosen small enough, $\Psi^{\prime}(x, \epsilon)$ must vanish at a point $x=\xi,\left|\xi-x_{0}\right|<\delta_{2}$. If $\delta_{2} \leqq \delta$ and $\delta_{1} \leqq \Delta$ then $\Psi(\xi, \epsilon)=F(\xi)$ and also $\Psi(\xi, \epsilon)=G(\xi)$ and thus $\Psi(x, \epsilon)=\Phi(x, \epsilon)$.

Massachusetts Institute of Technology 